Article published in J. Mater. Sci. DOI 10.1007/s10853-016-0351-4

\title{
Slip dislocation and twin nucleation mechanisms in hep metals
}

\author{
Andriy Ostapovets ${ }^{1 *}$ and Anna Serra ${ }^{2}$ \\ ${ }^{1}$ Central European Institute of Technology - Institute of Physics of Materials (CEITEC IPM), \\ Academy of Sciences of the Czech Republic, Žižkova 22, 61662 Brno, Czech Republic \\ ${ }^{2}$ Department of Civil \& Environmental Engineering, Universitat Politecnica de Catalunya, \\ Jordi Girona 1-3, 08034 Barcelona, Spain
}

${ }^{*}$ Corresponding author: tel.: +420 532290 429, e-mail: ostapov@ipm.cz

\begin{abstract}
A new nucleation mechanism is proposed for $\{10 \overline{1} 1\}$ deformation twin in hep materials. The mechanism is based on the results of atomistic computer simulations. It was found that under high shear stress applied on $\{10 \overline{1} 1\}$ plane (the stress level is about $7 \%$ of shear modulus) the core of a slip dislocation can transform to a twin embryo. The transformation and subsequent twin growth is accompanied by nucleation and migration of interfacial defects including disconnections and stacking faults. The paper provides the analysis of the nature of these defects and describes the reactions between them.
\end{abstract}

Keywords: compression twinning, magnesium, nucleation, dislocation

\section{Introduction}

Twinning is a major component of deformation in some hep metals [1]. Twinning on $\{10 \overline{1} 1\}$ planes is often observed under compression parallel to the [0001] (or c) axis. The $\{10 \overline{1} 1\}$ twinning is observed, for instance under high compression, e.g. $25 \%$ strain at room temperature [2], and under low compression at high temperature, above $400^{\circ} \mathrm{C}$ [3].In magnesium and its alloys $\{10 \overline{1} 1\}$ twins are less frequent than tensile $\{10 \overline{1} 2\}$ twins. However, they were observed, for instance, in AZ31 alloy under uniaxial compression of samples with basal texture [4].

Several twinning modes on $\{10 \overline{1} 1\}$ planes are possible. Two of them are traditionally taken into account. The first one has twinning direction along $<10 \overline{1} 2>$. This is the twinning mode, which is normally observed under deformation. The other one has twinning direction with irrational indices and it is important for theory of martensitic transformations. Despite the difference in the growth mechanisms of these modes, the final orientation of twin relative to the matrix is the same in both cases. Consequently, the modes are indistinguishable from crystallographic point of view. The study of $\{10 \overline{1} 1\}$ twins has a long history. The interfacial structure of $\{10 \overline{1} 1\}$ twins, obtained using atomic-scale computer simulation by Serra et al. in 1991 [5], is consistent with high-resolution transmission electron micrographs (HRTEM) of such twins in Ti obtained by Kasukabe et al. in 1993 [6, 7]. Thus, two different twinning dislocations were identified in [7] and their Burgers vectors, named $b_{2 / 2}$ and $b_{4 / 4}$,were further analyzed in [8] using the crystallography and topological characterization of twinning dislocations in h.c.p. metals discussed in [9]. The same twin dislocations $\left(b_{2 / 2}\right.$ and $\left.b_{4 / 4}\right)$ were analyzed in [10] and reasonable agreement with the predicted structures was obtained at about $0.17 \mathrm{~nm}$ resolution. The structures and energies of compression twin boundaries in hep Ti were studied by ab initio calculations in [11] and obtained by HRTEM in 
$[12,13]$ confirming the previous results. The interaction of a screw $<\mathrm{a}>$ dislocation with the twin boundary was studied in $[14,15]$. It was found that there is full absorption of the dislocation with the creation of a dipole of $b_{2 / 2}$ twinning dislocations. Recently Barrett and El Kadiri [16] reported the impact of deformation faceting on the $\{10 \overline{1} 1\}$ embryonic twin nucleation. Twin nucleation mechanisms by dissociation of bulk dislocation were also analyzed in the literature [17,18]. A comprehensive analysis of such processes by means of continuum elasticity was performed in [18]. It was concluded that the dissociation of a single dislocation is not likely to lead to a stable twin fault loop regardless of the applied stress, twin fault energy and length of slip segment.

In the present paper, we report a nucleation mechanism for $\{10 \overline{1} 1\}$ twinning in magnesium found by computer simulation. The mechanism is based on the transformation of the core of a single $<\mathrm{a}>$ dislocation into a twin nucleus under high applied stress. The proposed mechanism is similar to one of the mechanisms discussed in [18]. However, the atomistic simulations enable the real dislocation core structure to be taken into account and demonstrate the feasibility of the dissociation. We provide the analysis of the transformation mechanism as well as the analysis of the interfacial defects taking part in the nucleation and subsequent twin growth.

\section{Methods}

The calculations were performed using the embedded atom method with the potential developed by Sun et. al [19] and employing LAMMPS software [20]. OVITO [21] was used for visualization. The simulation block was a cylinder with radius of $240 \AA$. The $z$-axis (i.e. axis of cylinder) was aligned with the [1 $1 \overline{2} 10]$ direction. The $x$-axis was aligned with [ $\overline{1} 012]$ and $y$-axis was along the normal to $\{10 \overline{1} 1\}$ plane.

A screw dislocation with dislocation line along [1릴 direction was inserted in the center of the simulation box by imposing the anisotropic elastic field obtained from the analytical solution [22]. Periodic boundary conditions were applied in $z$-direction. Positions of atoms in the outer layer of the block were fixed in $x$ - and $y$-directions and energy minimization was performed in the inner part of the cylinder in order to obtain a relaxed dislocation core. Several types of $<\mathrm{a}>$ dislocation cores are possible [23-25] in hcp metals, being either dissociated or compact. Both types of core were found in our simulations. Structures of these cores are shown in Fig. 1. They are in agreement with previously reported results for Sun et al. potential [25]. The core is dissociated in basal plane (Fig.1a) if the dislocation glides along this plane. This is the classical dissociation into two partials with the Burgers vectors $1 / 3[10 \overline{1} 0]$ and $1 / 3[01 \overline{1} 0]$ connected by a stacking fault ribbon. The stacking fault is visible in Fig. 1a as the region with fcc structure (green atoms). However, such cores cannot glide along prismatic $\{10 \overline{1} 0\}$ planes. Cross slip to a prismatic plane leads to conversion of the core into the compact form (Fig. 1b). This compact core is spread on prismatic plane allowing dislocation glide along this plane. In principle such core can be also treated as dissociated on prismatic plane [23]. However, we use here the term "compact" because the partials are very close to each other in our case and cannot be confidently distinguished. The two core types can be obtained by insertion of the elastic field center at different positions relative to the unit cell. In addition, mutual transformations between dissociated basal core and compact prismatic core take place during cross slip under applied stress. However, we limit our consideration to the compact core in the present paper. We found that this type of core can serve as a nucleation place for twins. This does not imply that basal core dissociation cannot lead to twin nucleation but, it is likely that basal core transforms to compact form before twin nucleation.

The evolution of dislocation core was studied by the application of strain to the block in small steps and subsequent minimization of block energy by the conjugate gradient method. 

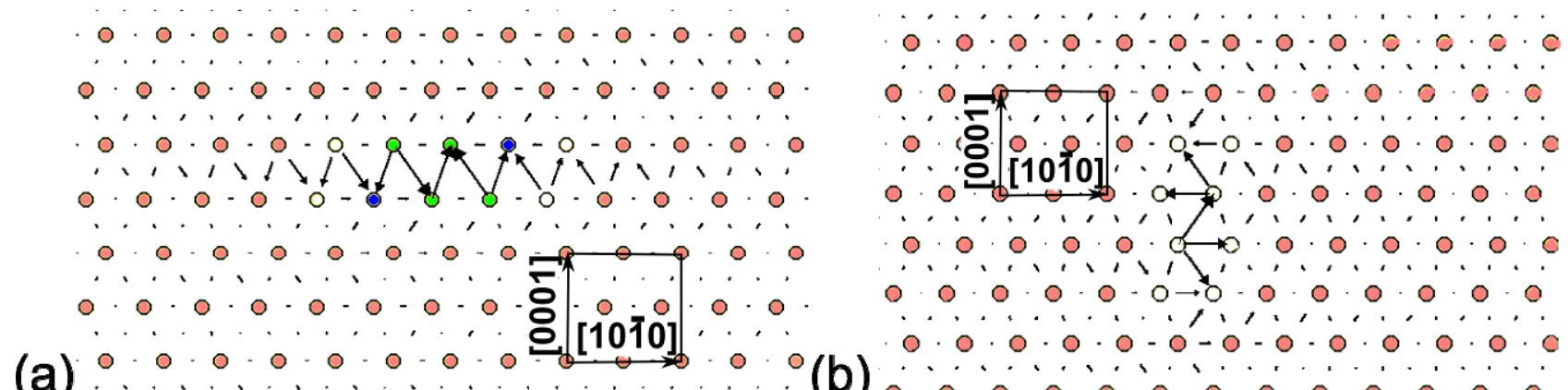

Fig.1. Structures of screw $\langle a\rangle$ dislocation cores: (a) core dissociated in basal plane and (b) compact core spread in prismatic plane. The projection is along the $\langle 1 \overline{2} 10\rangle$ direction. The coloring is applied according to the lattice type: pink - hcp, green - fcc, blue - bcc, white - undefined. The arrows are proportional to screw components of relative displacements.

\section{Results and discussion}

\section{Twin nucleation}

Fig. 2 shows the evolution of a dislocation core under an applied shear stress in $\{10 \overline{1} 1\}$ shear plane. The initial compact core (Fig.2a) is spread along $\{10 \overline{1} 1\}$ plane after the application of a $\sigma_{x y}$ stress component to the block (Fig.2b). However, this stress component alone is not sufficient to stimulate twin nucleation. Increasing $\sigma_{x y}$ even up to $0.2 \mathrm{G}$, where $\mathrm{G}$ is shear modulus, leads neither to glide along the plane nor to further dissociation. However, the application of $\sigma_{\mathrm{zx}}$ stress simultaneously with $\sigma_{\mathrm{xy}}$ allows the dissociation and subsequent transformation of core to the twin embryo. We found that the necessary values of stress are $\sigma_{\mathrm{xy}}=1360 \mathrm{MPa}(0.07 \mathrm{G})$ and $\sigma_{\mathrm{zx}}=285 \mathrm{MPa}$ $(0.014 \mathrm{G})$.These values are much higher than the resolved shear stresses for basal (3.6 MPa) and prismatic (44 MPa) slip [25]. Figs. 2(c-h) demonstrate the subsequent evolution of the dislocation core. In the first stages the core begins to move along $\{10 \overline{1} 1\}$ plane and spreads a bit along this plane (Fig 2c). Simultaneously it becomes zig-zag shaped with 4-layer height segments on the left side and 2-layer height segments on the right side of the core (Fig.2d). The next stage of perturbation leads to the development of a clearly visible twin embryo on the left side of core (Fig.2e). This embryo has 4-layer height. The right edge is significantly distorted and continues its migration. Three step-like defects occur in the surface of the twin embryo behind of the migrating right edge. These defects remain sessile during twin growth. They are marked by arrows in Fig. $2 f$. The twin propagates mainly to the right side at this stage. Then the twin growth continues by nucleation of disconnection dipoles. Such dipoles are marked by arrows in Fig. $2 \mathrm{~g}$. One disconnection of the dipole glides to the right along the twin boundary. This migration mediates twin growth in the direction perpendicular to the $\{10 \overline{1} 1\}$ plane (Figs.2g,h). It is worth noting that the one-layer step defects do not serve as obstacles to disconnection motion (Fig.2h) 
(a)

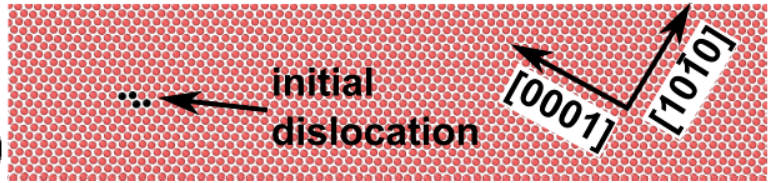

(c)

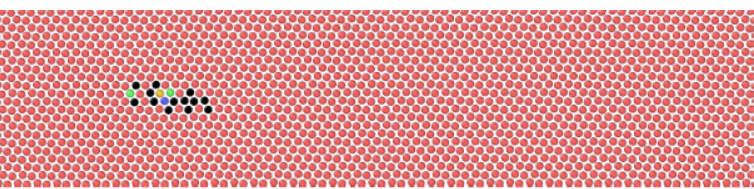

(e)
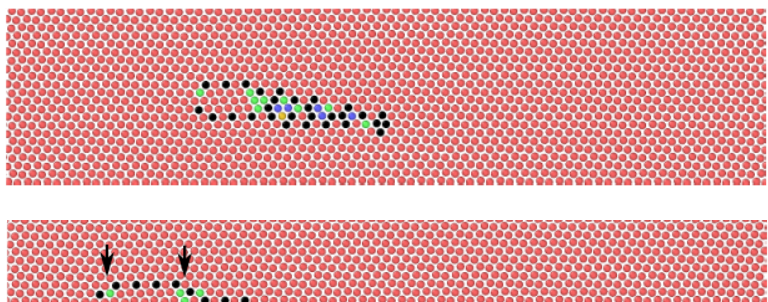

(g)

(d)

(b)

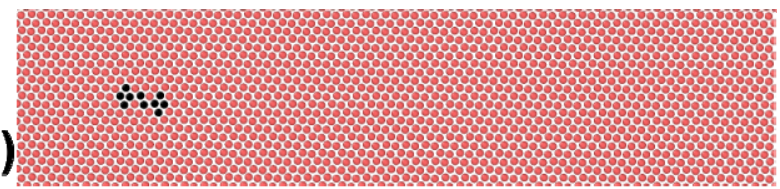

(f)

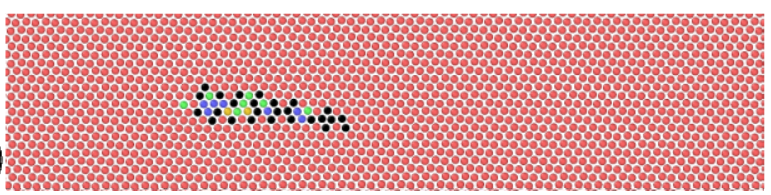

\section{)}

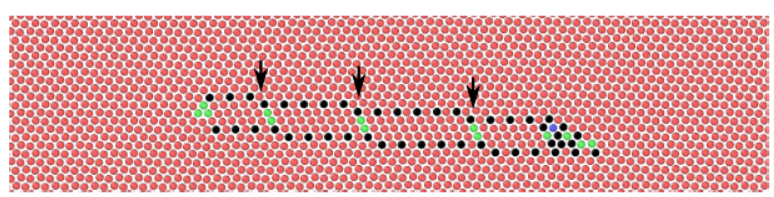

(h)

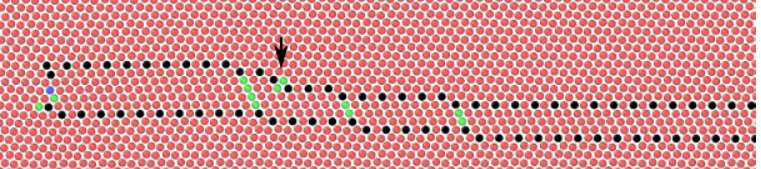

Fig. 2. Development of a $\{10 \overline{1} 1\}$ twin from a single $<a>$ screw dislocation. Projection along the $\langle 1 \overline{2} 10\rangle$ direction. The coloring is applied according to the lattice type: pink-hcp, green-fcc, blue - bcc, black-undefined.

\section{Analysis of observed interfacial defects}

We demonstrated above that the dissociation of dislocation core can lead to the formation of a twin embryo. In this section we study details of the dissociation. We analyze Burgers circuits around defects in the twin embryo in order to determine their nature.

Let us recall the most important disconnections for $\{10 \overline{1} 1\}$ twinning. According to Ref. [5] they have the following Burgers vectors:

$$
\begin{gathered}
\boldsymbol{b}_{2 / 2}=\frac{1}{\left(6 L^{2}+3\right)}\left\langle 5-2 L^{2},-\left(2 L^{2}+1\right), 4\left(L^{2}-1\right), 3\left(2 L^{2}-3\right)\right\rangle \\
\boldsymbol{b}_{4 / 4}=\frac{\left(2 L^{2}-3\right)}{\left(2 L^{2}+1\right)}\langle\overline{1} 012\rangle,
\end{gathered}
$$

where $L=\frac{c}{a} \sqrt{\frac{2}{3}}$. The magnitudes of these vectors are

$$
\begin{gathered}
\left|\boldsymbol{b}_{2 / 2}\right|=\frac{a \sqrt{\left(16(c / a)^{6}-56(c / a)^{4}+33(c / a)^{2}+63\right.}}{\sqrt{3+4(c / a)^{2}}} \\
\left|\boldsymbol{b}_{4 / 4}\right|=\frac{a\left(4(c / a)^{2}-9\right)}{\sqrt{3+4(c / a)^{2}}}
\end{gathered}
$$


The magnitudes given by (3) and (4) are $0.542 a$ and $0.421 a$, respectively, for lattice parameters $(a=3.203 \AA, c / a=1.624)$ of magnesium. It is worth to note that $\boldsymbol{b}_{2 / 2}$ is responsible for the propagation of a twinning mode with irrational twinning direction. Consequently, $\boldsymbol{b}_{2 / 2}$ is not aligned with the rational [1012] twinning direction. The Burgers vector of $\boldsymbol{b}_{2 / 2}$ can be decomposed into screw and edge parts if the dislocation line is along [1 $\overline{2} 10]$ direction:

$$
\boldsymbol{b}_{2 / 2}=\boldsymbol{b}_{2 / 2}^{s}+\boldsymbol{b}_{2 / 2}^{e}
$$

where the screw part is $\pm 1 / 6[1 \overline{2} 10]$. Another important relation is

$$
\boldsymbol{b}_{4 / 4}=2 \boldsymbol{b}_{2 / 2}^{e}
$$

i.e. $\boldsymbol{b}_{4 / 4}$ disconnection can be considered as the sum of two $\boldsymbol{b}_{2 / 2}$ with opposite screw components.

Fig. 3 shows the dichromatic complex for $\{10 \overline{1} 1\}$ twin boundary. The Burgers vectors of $\boldsymbol{b}_{2 / 2}$ and $\boldsymbol{b}_{4 / 4}$ disconnections are shown. It can be seen that besides $\boldsymbol{b}_{2 / 2}$ disconnection with upward step in the boundary, there is a $\boldsymbol{b}_{-2 /-2}$ disconnection with downward step. These disconnections have opposite edge components but they could have the same screw component, if so their addition is an $<a>$ screw dislocation. Conversely, when a screw dislocation intersects the twin boundary it decomposes into $\boldsymbol{b}_{2 / 2}$ and $\boldsymbol{b}_{-2 /-2}[14,15]$.

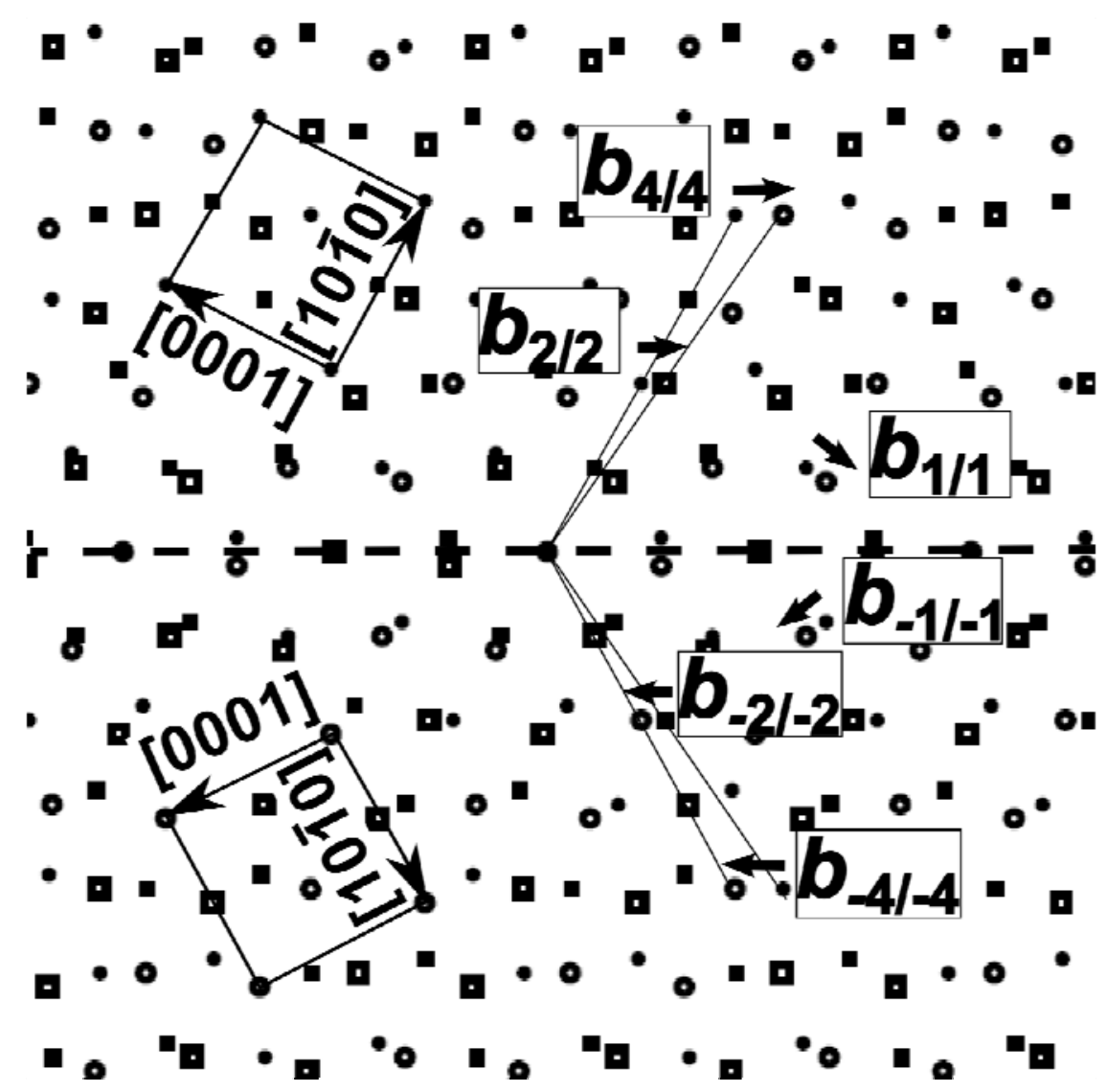

Fig. 3. Dichromatic complex for $\{10 \overline{1} 1\}$ twin projected along $\langle 1 \overline{2} 10\rangle$ direction. Burgers vectors of possible disconnections are marked by arrows. Hexagonal unit cells in black and white crystals are shown by rectangles. The black and white crystals correspond to matrix and twin respectively. 
Several types of defects are observed in the twin boundaries in our simulations. They are twin tips (Fig. 4 and Fig. 5a) and step-like disconnections in the $\{10 \overline{1} 1\}$ boundaries (Fig.5b,c). These defects are shown in Figs. 4 and 5 together with the Burgers circuits used for the analysis. Two types of disconnections are found, i.e., two layer step disconnections in the flat $\{10 \overline{1} 1\}$ boundary and one layer step disconnections $\left(\boldsymbol{b}_{1 \mathrm{sf}}\right)$ at the intersections of $\{10 \overline{1} 1\}$ boundaries with basal stacking faults (Fig.5c).

The $\{10 \overline{1} 1\}$ plane is an invariant plane for the twinning shear deformation. Consequently, the perfect twin boundary is free of defects. However, it is possible to ascribe some Burgers vector for the twin tip. The tip accommodates discrepancy between a real finite twin boundary and the matrix in contrast to the case of ideal infinite twin. In the latter case the boundary lies only on the invariant plane so good fit between matrix and twin is reached. One can also treat the twin as multilayer stacking fault. The dislocations residing at twin tips are analogous to a certain extent to the partials which terminate stacking faults inside a crystal.

The Burgers circuit around twin tip is shown in Fig. 4a. The sign of $b$ is consistent with the RH/FS convention with positive line sense into the paper (away from reader) and clockwise circuit direction. Fig. $4 \mathrm{~b}$ shows the same circuit in the dichromatic pattern, where the nature of the closure failure is better seen. The analysis of this circuit leads to the conclusion that the Burgers vector of the left tip is equal to $\boldsymbol{b}_{4 / 4}$. However, analysis of atomic coordinates in the simulation block shows that the circuit in Fig. 5a includes an element out of the plane of paper due to the presence of a screw component. Unfortunately, this fact is difficult to show in projection. The total Burgers vector of the right tip (Fig. 5a) is equal to $-\boldsymbol{b}_{4 / 4}+<\mathbf{a}>$, i.e. substantial part of initial dislocation is settled here.
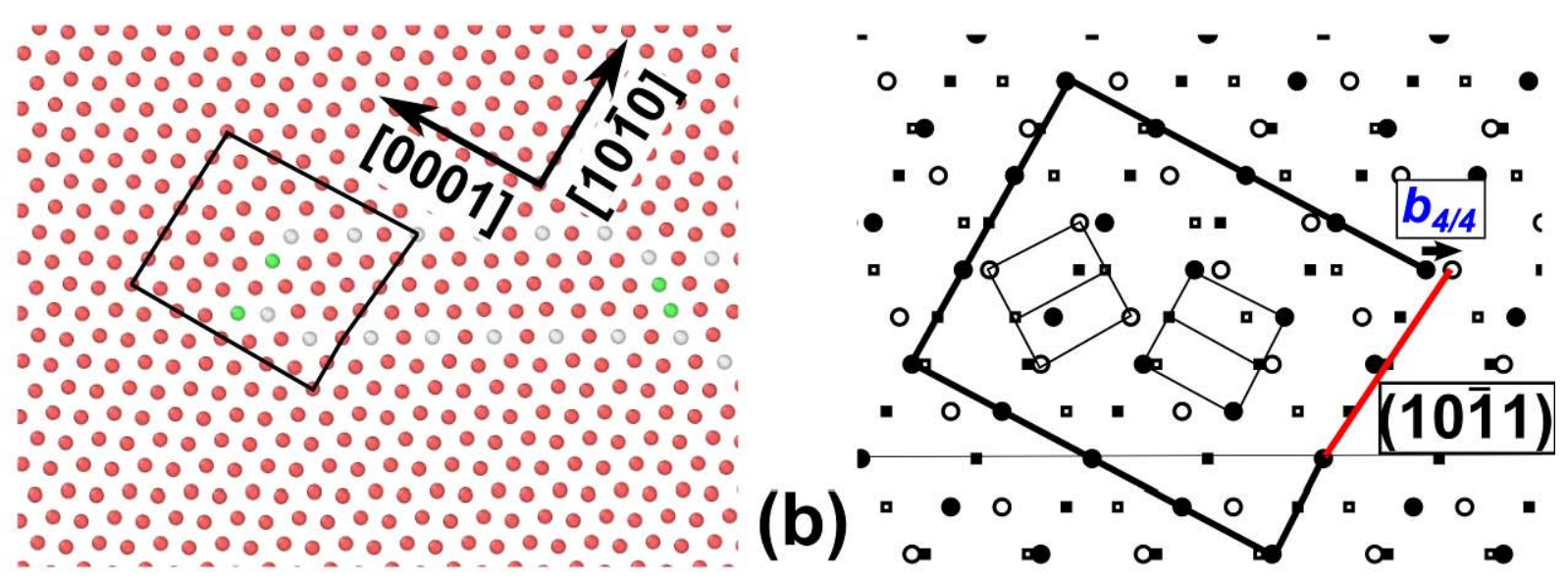

Fig.4. (a) Burgers circuit around left side of the twin tip in simulated configuration, (b) the same circuit after mapping into the dichromatic pattern.Projection along $\langle 1 \overline{2} 10\rangle$ direction.

The Burgers vectors of sessile $\boldsymbol{b}_{1 \text { sf }}$ defects can be obtained from the analysis of circuits in Fig. 5c; they are equal to

$$
\boldsymbol{b}_{1 s f}=\frac{1}{\left(6 L^{2}+3\right)}\left\langle L^{2}-2,2\left(L^{2}-2\right),-3\left(L^{2}-2\right), 0.5\left(6 L^{2}-7\right)\right\rangle
$$




$$
\left|\boldsymbol{b}_{1 s f}\right|=\frac{a \sqrt{36(c / a)^{4}-141(c / a)^{2}+144}}{6 \sqrt{3+4(c / a)^{2}}}
$$

The length of $\boldsymbol{b}_{1 \mathrm{sf}}$ vector is $0.215 a$ for magnesium.

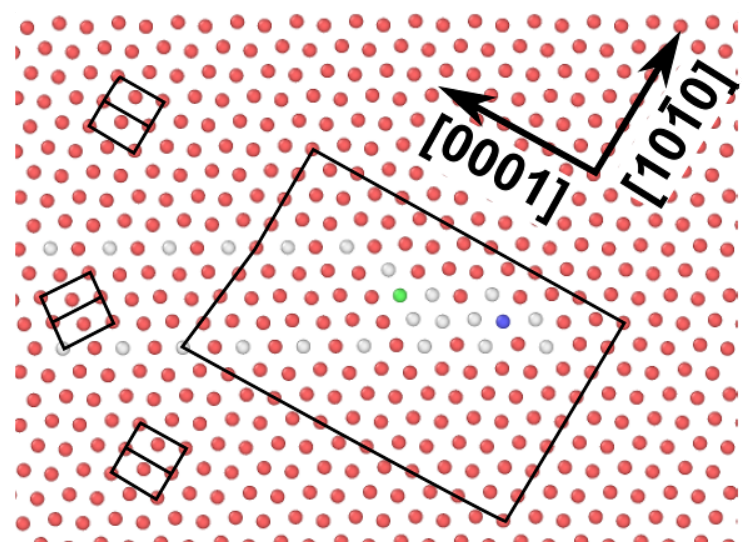

․․

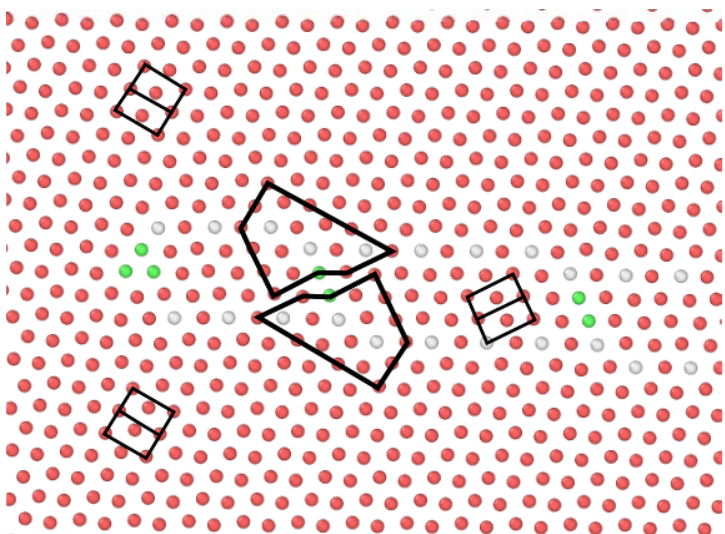

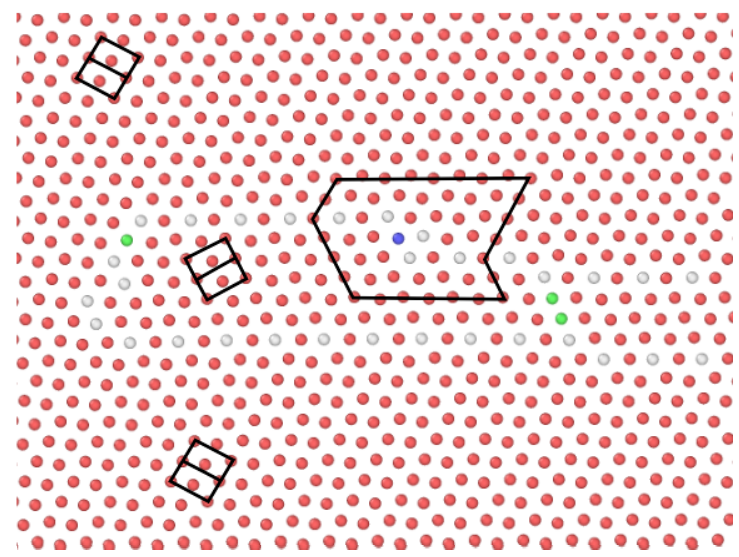

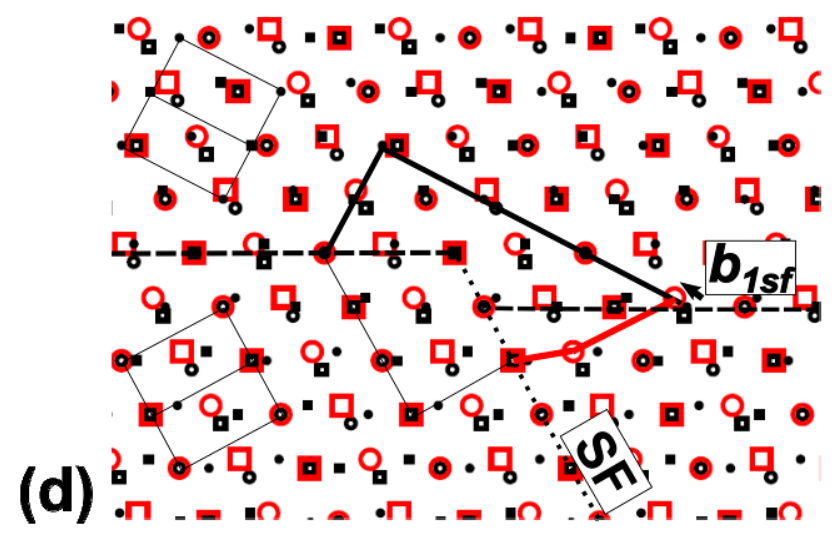

Fig.5. Burgers circuits around crystallographic defects in twin nucleus: (a) right twin edge, (b) $\boldsymbol{b}_{-2 /-}$ 2 disconnection in twin boundary, (c) closed circuit around a $b_{1 s f}$ defect, (d) circuit in (c) after mapping into a trichromatic complex. White and red complexes correspond to twin crystals with different basal stacking sequences (...ABAB... for white and ...BCBC... for red) and black complex corresponds to matrix. The Burgers circuit in (d) corresponds to the upper circuit in (c). Projection in $\langle 1 \overline{2} 10\rangle$ direction.

The $\boldsymbol{b}_{1 \text { sf }}$ defect delineates the junction between twin boundary segments and an $\mathrm{I}_{1}$ type stacking fault. Fig. 5d reproduces the upper Burgers circuit from Fig. $5 \mathrm{c}$ in a trichromatic complex. The trichromatic complex shows overlapping lattice complexes of the crystals in different sides of the boundaries. Such construction is useful for the analysis of defects in boundary junctions [26]. The black atoms correspond to the matrix (crystal above the twin boundary), white atoms correspond to twin laying to the left of the stacking fault and red atoms correspond to the twin lying to right of the stacking fault. The white and red crystals have different basal stacking sequences. The closure failure for the Burgers circuit corresponds to the Burgers vector $\boldsymbol{b}_{1 \text { sf }}$ defined by Eq. (7) and its length is given by Eq. (8). This Burgers vector can be also understood as the combination of a step due to $<\mathrm{c}>$-dislocation in the matrix and a near-complementary step produced by $<$ a $>$ dislocation combined a stacking fault partial in the twin. In this case the $\boldsymbol{b}_{1 \text { sf }}$ can be presented as 
$\boldsymbol{b}_{1 \mathrm{sf}}=\boldsymbol{t}_{\lambda}-\boldsymbol{t}_{\mu}, \quad$ where $\boldsymbol{t}_{\lambda}=1 / 3[11 \overline{2} 0]+1 / 6[2 \overline{2} 03] \quad$ and $\boldsymbol{t}_{\mu}=[0001] . \quad$ The component $1 / 6[2 \overline{2} 03]$ corresponds to the partial which terminates an $\mathrm{I}_{1}$ stacking fault obtained from an E type fault by shear [22].

The sum of the Burgers vectors of $\boldsymbol{b}_{1 \text { sf }}$ type defects in the simulation block is 0 . The defects are similar to those observed in the simulations of Pinsook and Ackland [27-29] by using an interatomic potential for zirconium. Pinsook and Ackland did not analyze the Burgers vectors of these defects. However, their observations support our view that the presence of $\boldsymbol{b}_{1 \text { sf }}$ defects in our simulations is not an artifact due to the potential used. The $\boldsymbol{b}_{1 \mathrm{sf}}$ defects occur during twin nucleation and are not the result of the interaction of the twin boundary with the stacking fault propagating in the crystal. It is also worth noting that Burgers vector of $\boldsymbol{b}_{1 \text { sf }}$ is not equal to Burgers vector of $\boldsymbol{b}_{-1 / 1}$ disconnection marked in Fig. 3. This difference is due to the presence of the basal stacking fault connected to the interfacial defect.

The last kind of defects found in the twin boundaries are two layer steps (Fig. 5b). The Burgers circuit analysis leads to the conclusion that the Burgers vectors of such defects are of the $\boldsymbol{b}_{2 / 2}$ type defined by Eq. (1). The $\boldsymbol{b}_{2 / 2}$ disconnections are nucleated in pairs $\boldsymbol{b}_{2 / 2}$ and $-\boldsymbol{b}_{2 / 2}$ during the growth of twin. It was found that the mechanism is the same as reported in [30], i.e. subsequent nucleation of $\boldsymbol{b}_{2 / 2}$ pairs with opposite signs of screw components is observed. Due to relations (5) and (6), the migration of two $\boldsymbol{b}_{2 / 2}$ disconnections with opposite screw components is equivalent to the migration of a $\boldsymbol{b}_{4 / 4}$ disconnection. The Burgers vector content of twin tips increases during twin growth due to the mutual interaction of incoming $\boldsymbol{b}_{2 / 2}$ disconnections.

\section{Discussion}

The observed reaction of twin nucleation through dissociation of a screw $<\boldsymbol{a}>$ dislocation can be written as follows:

$$
<\boldsymbol{a}>=\left(<\boldsymbol{a}>-\boldsymbol{b}_{4 / 4}\right)+\boldsymbol{b}_{4 / 4}+3 \boldsymbol{b}_{1 s f}-3 \boldsymbol{b}_{1 s f}
$$

This reaction is similar to the following reaction discussed in [18] on the basis of continuum linear elasticity:

$$
<\boldsymbol{a}>=\left(<\boldsymbol{a}>-\boldsymbol{b}_{4 / 4}\right)+\boldsymbol{b}_{4 / 4}
$$

However, it was concluded in [18] that reaction (10) does not lead to the stable twin for simply increased applied stress. Atomistic simulations allow us to take into account finer details of dislocation core than the elastic theory. It is probably the reason for the different predictions of these two models. The sessile defects $\boldsymbol{b}_{1 \text { sf }}$ seems to be a part of the nucleation process and they are not simply the result of complex stress state. They occur in the initial stages of nucleation and no new defects of such type occur during subsequent growth in our simulations. In our opinion, the presence of $\boldsymbol{b}_{1 \text { sf }}$ decrease the attraction between twin embryo tips allowing an easier development of the twin embryo.

Let us also consider some crystallographic details which can be important for the process of twin nucleation. The slip $\langle\boldsymbol{a}\rangle$ dislocation is normally dissociated in the basal plane. However, a possible dissociation on the $\{10 \overline{1} 1\}$ plane can be considered by the analysis of $\{10 \overline{1} 1\} \Gamma$-surface. The $\Gamma$-surface is obtained by cutting the crystal in the crystallographic plane and subsequent shift of one part relative to the other one. The dependence of the obtained fault energy on the shift is the $\Gamma$ surface. The $\{10 \overline{1} 1\} \Gamma$-surface is shown in Fig.6, where the Burgers vector of $<\boldsymbol{a}>$ slip dislocation is 
shown by a black arrow and the Burgers vectors of possible partials are shown by yellow arrows. A possible dissociation can be proposed as

$$
\boldsymbol{b}_{\dot{a}}=\boldsymbol{b}_{s f 1}+\boldsymbol{b}_{s f 12}+\boldsymbol{b}_{s f 21}-\boldsymbol{b}_{s f 1}
$$

It is worth noting that the length of the edge component of $\boldsymbol{b}_{\text {sf12 }}$ can be estimated as

$$
\left|\boldsymbol{b}_{s f 12}^{e}\right|=\frac{3 a}{2 \sqrt{3+4(c / a)^{2}}}
$$

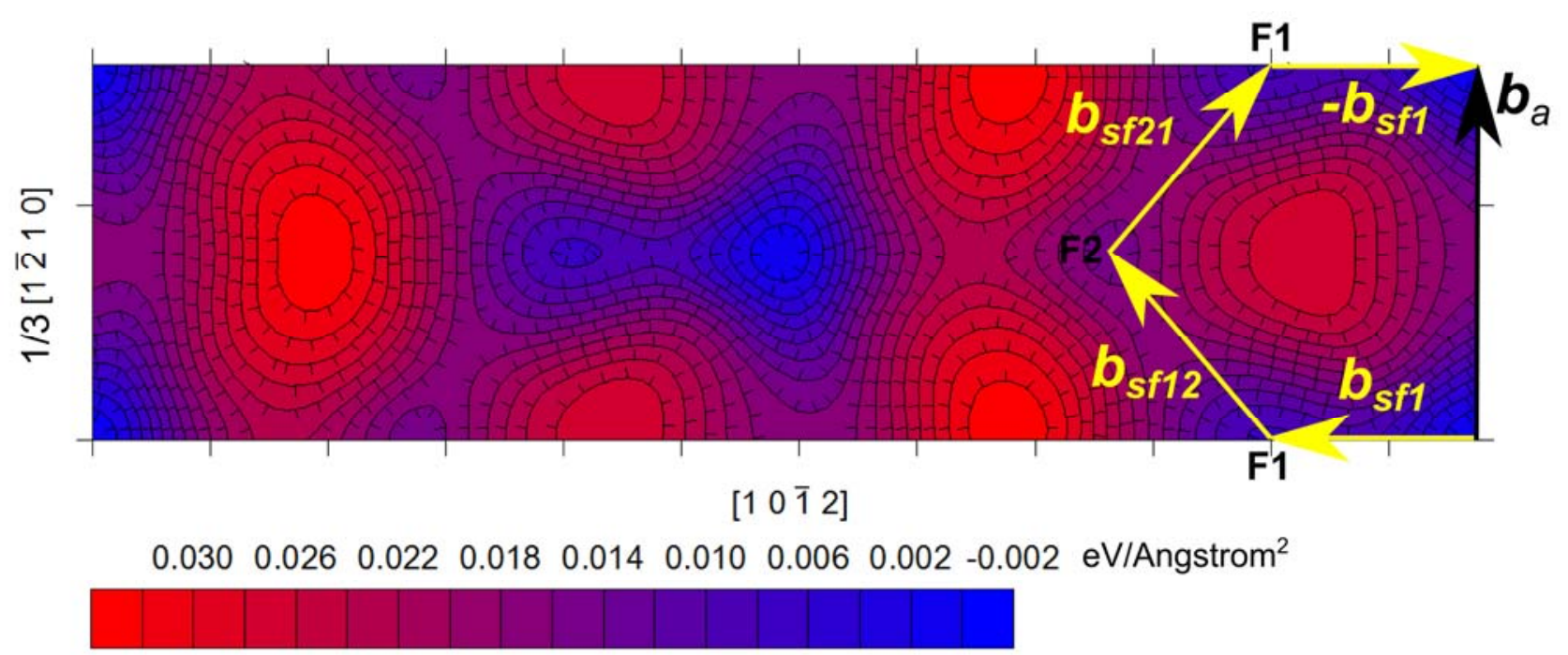

Fig.6. $\{10 \overline{1} 1\} \Gamma$-surface. The Burgers vector of a perfect $<a>$ dislocation is shown by the black arrow. Possible splitting into partial dislocations is shown by yellow arrows.

It can be seen that (12) and (4) are equal for $\mathrm{c} / \mathrm{a}=1.620$, which is close to $\mathrm{c} / \mathrm{a}$ of magnesium (1.624) and to c/a of Sun potential (1.628). The magnitude represented in (12) is equal to $0.408 a$ for magnesium. Consequently, the Burgers vector of twinning disconnection can occur in the process of dislocation core dissociation. However, dissociation according to (11) is not energetically favorable at normal conditions. Similar reaction can take place only under high shear stress on $\{10 \overline{1} 1\}$ plane. Although reaction (11) is not identical to (9) or (10), it may be relevant to the nucleation event.

\section{Conclusions}

Nucleation of a $\{10 \overline{1} 1\}$ twin by dissociation of a screw $<a>$ dislocation is observed in atomistic simulations of magnesium. The nucleation takes place at a relatively high level of applied stress (0.07G).

The observed dissociation is similar (but not identical) to those previously discussed in terms of continuum elastic theory. Atomistic simulations provide a more detailed description of core structure by comparison with continuum elastic theory. The proposed dissociation reaction is

$$
<\boldsymbol{a}>=\left(<\boldsymbol{a}>-\boldsymbol{b}_{4 / 4}\right)+\boldsymbol{b}_{4 / 4}+3 \boldsymbol{b}_{1 s f}-3 \boldsymbol{b}_{1 s f}
$$

The presence of $\boldsymbol{b}_{1 \mathrm{sf}}$ at the initial stage facilitates development of the twin embryo. 
In contrast to the prediction of elastic theory, our atomistic simulations show that twin nucleation from an individual dislocation is possible at a sufficiently high value of the applied stress.

\section{Acknowledgements}

Financial Supports from Czech Science Foundation (project 16-14599S), from the Spanish MINECO (FIS2015-69017-P) and Ministry of Education, Youth and Sports of the Czech Republic under project CEITEC 2020 (LQ1601) are gratefully acknowledged.

Conflict of Interest: The authors declare that they have no conflict of interest.

\section{References}

[1] Christian JW, Mahajan S (1995) Deformation twinning. Prog Mater Sci 39:1-157

[2] Komninou P,Kehagias T, Karakostas T, Antonopoulos JG, Braisaz T, Nouet G, Serra A (2000) Electron Microscopy of Defects in Hexagonal Materials. In: Lepinoux J et al (Eds.) Multiscale Phenomena in Plasticity, Kluwer, Boston, pp. 215-226

[3] Paton NE, Backofen WA (1970) Plastic Deformation of Titanium at Elevated Temperatures.Metall Trans 1:2839-2847

[4] Meng L, Yang P, Xie Q, Mao W (2008) Analyses on compression twins in magnesium. Mater Trans 49:710-714

[5] Serra A, Pond RC, Bacon DJ (1991) Computer simulation of the structure and mobility of twinning dislocations in H.C.P. metals. Acta Metall Mater 39:1469-1480

[6] Kasukabe Y, Yamada Y, Peng JL, Bursill LA (1993) Structure analysis of $\{1 \overline{1} 01\}$ twins in evaporated titanium thin films. Phil. Mag. A 68:587-598

[7] Kasukabe Y, Yamada Y, Peng JL, Bursill LA (1993) Characterization of $\{1 \overline{1} 01\}$ twin dislocation structures in evaporated titanium thin films by high-resolution transmission electron microscopy. Phil Mag Lett 67:361-368

[8] Pond RC, Bacon DJ, Serra A (1995) Interfacial structure of $\{10 \overline{1} 1\}$ twins and twinning dislocations in titanium. Phil Mag Lett 71:275-284

[9] Serra A, Bacon DJ, Pond RC (1988) The crystallography and core structure of twinning dislocations in hep metals. ActaMetall 36:3183-3203

[10] Bursill LA, Peng JL, Fan XD, Kasukabe Y, Yamada Y (1995) $\{10 \overline{1} 1\}$ twin dislocation structures in evaporated titanium thin films. Phil Mag Lett 71:269-273

[11] Morris JR, Ye YY, Ho M, Chan CT, Yoo MH (1995) Structures and energies of compression twin boundaries in hep Ti and Zr. Phil Mag A 72:751-753

[12] Wang YC, Ping DH, Li DX, Ye HQ (1996) A high-resolution transmission electron microscopy study of the $\{10 \overline{1} 1\}$ twin-boundary structure in alpha-Ti. Phil Mag Lett 74:367-373 
[13] Braisaz T, Ruterana P, Nouet G, Komninou P, Kehaigas T, Karakostas T, Poulopoulos P, Aggelakeris M, Flevaris N, Serra A (1998) Nanocrystalline thin titanium films grown on potassium bromide single crystals. Thin Solid Films 19:140-143

[14] Serra A, Bacon DJ (1995) Computer simulation of screw dislocation interactions with twin boundaries in H.C.P. metals. Acta metall mater 43:4465- 4481

[15] Wang J, Beyerlein IJ, Hirth JP (2012) Nucleation of elementary $\{\overline{1} 011\}$ and $\{\overline{1} 013\}$ twinning dislocations at a twin boundary in hexagonal close-packed crystals. Model Sim Mater Sci Eng 20:024001

[16] Barrett CD, El Kadiri H (2014) Impact of deformation faceting on $\{10 \overline{1} 2\},\{10 \overline{1} 1\}$ and $\{10 \overline{1} 3\}$ embryonic twin nucleation in hexagonal close-packed metals. Acta Mater 70:137-161

[17] Mendelson S (1969) Zonal Dislocations and Twin Lamellae in h.c.p. Metals.Mater SciEng 4:231-242

[18] Capolungo L, Beyerlein IJ (2008) Nucleation and stability of twins in hcp metals.Phys Rev B 78:024117

[19] Sun DY, Mendelev MI, Becker CA, Kudin K, Haxhimali T, Asta M (2006) Crystal-melt interfacial free energies in hcp metals: A molecular dynamics study of Mg. Phys Rev B 73:024116

[20] Plimpton S (1995) Fast Parallel Algorithms for Short-Range Molecular Dynamics. J Comp Phys 117:1-19

[21] Stukowski A(2010) Visualization and analysis of atomistic simulation data with OVITO-the Open Visualization Tool. Modell Simul Mater Sci Eng 18:015012

[22] HirthJP, Lothe J (1968) Theory of dislocations.McGraw-Hill, New York

[23] Vitek V (1992) Structure of dislocation cores in metallic materials and its impact on their plastic behavior. Progr. Mater. Sci. 36:1-27

[24] Serra A, Bacon B (1995) Computer simulation of screw dislocation interactions with twin boundaries in h.c.p. metals. Acta Metall. Mater. 43:4465-4481

[25] Yasi JA, Nogaret T, Trinkle DR, Qi Y, Hector Jr LG, Curtin WA (2009) Basal and prism dislocation cores in magnesium: comparison of first-principles and embedded-atom-potential methods predictions.Modell Simul Mater Sci Eng17:055012

[26] Barrett CD, El Kadiri H (2014) Fundamentals of mobile tilt grain boundary faceting. Scripta Mater 84-85:15-18

[27] Pinsook U, Ackland GJ (1998) Simulation of martensitic microstructural evolution in zirconium.Phys Rev B 58:11252-11257

[28] Pinsook U, Ackland GJ (2000) Atomistic simulation of shear in a martensitic twinned microstructure.Phys Rev B 62:5427-5434

[29] Ackland GJ (2000) Simulations of martensitic microstructure. J Mater Sci 40:3205-3208

[30] Wang J, Beyerlein IJ, Hirth JP, Tome CN (2011) Twinning dislocations on $\{10 \overline{1} 1\}$ and $\{10 \overline{1} 3\}$ planes in hexagonal close-packed crystals. Acta Mater 59:3990-4001 